\title{
Timing Observations of PSR B1937+21 at CRL
}

\author{
Y. Hanado, Y. Shibuya, F. Nakagawa, M. Sekido, M. Hosokawa, M. \\ Imae
}

Communications Research Laboratory (CRL), 4-2-1, Nukui-Kita, Koganei, Tokyo 184-8795, Japan

\begin{abstract}
Pulsar parameters of PSR B1937+21 acquired from observations at the Kashima 34-m antenna over five years are consistent with published reports. The frequency stability is $2 \times 10^{-14}$ over the data span of 6.5 years using Kashima data combined with Parkes data.
\end{abstract}

\section{Introduction}

CRL has conducted investigations on a millisecond pulsar to apply them to a precise time scale. We developed a timing observation system which uses an AOS (Acousto-Optic Spectrometer) instead of filter bank and has $200 \mathrm{MHz}$ bandwidth (BW; $200 \mathrm{kHz} \times 256 \mathrm{ch} \times 4$ units). We started weekly observations of PSR $\mathrm{B} 1937+21$ at S-band with the $34-\mathrm{m}$ antenna at Kashima $\left(35^{\circ} 57^{\prime} \mathrm{N}, 140^{\circ} 39^{\prime} \mathrm{E}\right)$ in 1997 Nov. We have reported the pulsar's parameters and frequency stability by using one unit's data $(50 \mathrm{MHz} \mathrm{BW}$ ) for the first three years (Hanado et al. 2002 ). In this paper we report on data from four units (200 $\mathrm{MHz} \mathrm{BW})$, combined with Parkes data in order to verify that our data are consistent with other sites' data.

\section{Combination with Parkes Data}

We combined the Kashima data at S-band (1997 Nov-2003 Apr) with Parkes data at L-band (1995 Aug-1996 Sep), and carried out parameter fitting by using TEMPO (Taylor \& Weisberg 1989). Kashima data were obtained from one day's averaging and 4 units' accumulating. Initial parameters for fitting were derived from Lange et al. (2000), except DM deviation, which was newly estimated by the joint research with a Russian group (Ilyasov et al., these proceedings). The parameters after fitting (Table 1) were consistent with the previous reports (Kaspi et al. 1994). Figure 1a shows the residuals after fitting. Kashima residuals match well with Parkes's without any apparent gaps or unmodeled trends. Figure $1 \mathrm{~b}$ shows the frequency stability $\sigma_{z}$ (Matsakis et al. 1997) calculated from the residuals in Figure 1a. Our results are consistent with Arecibo's.

From these results, we can say that our data over 5 years are consistent with Parkes and other data. We were also able to check PSR B1937+21's frequency stability in the long term with this combination. These results are a good demonstration that a medium-aperture antenna is effective for timing observations of a strong millisecond pulsar. 
Table 1. Parameters for PSR B1937+21 obtained from Kashima data combined with Parkes data. DM is quoted from Lange et al. (2000), and DM deviation is quoted from Ilyasov et al. (these proceedings).

\begin{tabular}{|l|c||lr|}
\hline Parameters & Our fit results & & \\
\hline RA (J2000) & $19^{\mathrm{h}} 39^{\mathrm{m}} 38^{\mathrm{s}} .560107(7)$ & Time scale & UTC \\
Dec (J2000) & $21^{\circ} 34^{\prime} 59^{\prime} 1360(2)$ & Ephemeris & DE200 \\
PmRA (mas/yr) & $-0.04(4)$ & DM $\left(\mathrm{pc} \mathrm{cm} \mathrm{cm}^{-3}\right)$ & 71.0299 \\
PmDec (mas/yr) & $-0.53(7)$ & DM deviation & -0.0012 \\
$\pi(\mathrm{mas})$ & $2.9(8)$ & Epoch & MJD 51000 \\
$\nu(\mathrm{Hz})$ & $641.928249503944(2)$ & & \\
$\dot{\nu}\left(\mathrm{s}^{-2}\right)$ & $-4.33110(4) \times 10^{-14}$ & & \\
\hline
\end{tabular}

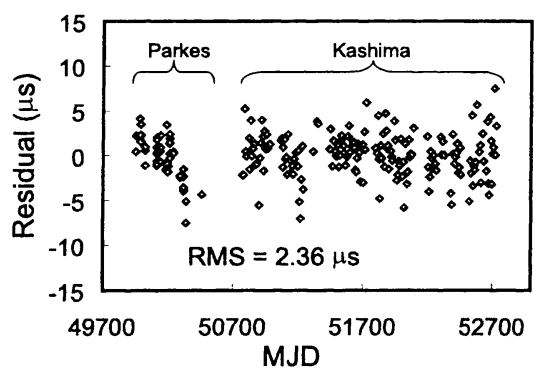

(a) Residuals

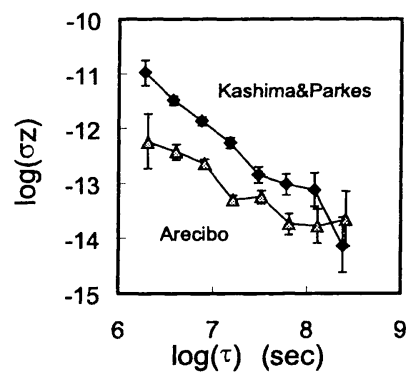

(b) Frequency Stability

Figure 1. (a) Post-fit timing residuals, and (b) frequency stability of PSR B1937+21 obtained from Kashima data combined with Parkes data. Arecibo data was quoted from Matsakis et al. (1997).

Acknowledgments. We would like to thank M. Bailes at Swinburne University of Technology for allowing us to use the Parkes data and for useful advice, and as well so many thanks to the Russian pulsar research group at P. N. Lebedev Physical Institute for their helpful advice.

\section{References}

Hanado, Y., Shibuya, Y., Hosokawa, M., Sekido, M., Gotoh, T., \& Imae, M. 2002, PASJ, 54, 305

Kaspi, V. M., Taylor, J. H., \& Ryba, M. F. 1994, ApJ, 428, 713

Lange, Ch., Wex, N., Kramer, M., Doroshenko, O., \& Backer, D. C. 2000, in ASP Conf. Ser., Vol. 202, Pulsar Astronomy - 2000 and Beyond, eds.

M. Kramer, N. Wex, \& R. Wielebinski, (San Francisco: ASP), p. 61

Matsakis, D. N., Taylor, J. H., \& Eubanks, T. M. 1997, A\&A, 326, 924

Taylor, J. H., \& Weisberg, J. M. 1989, ApJ, 345, 434 